\title{
7. Urinary Immunocytology and Bladder Carcinomas
}

\begin{tabular}{|l|l|l|}
\hline R. & Arndt \\
\hline H. & Huland \\
\hline
\end{tabular}

Department of Urology, University of Hamburg FRG

R. Arndt, Department of Urology, University of Hamburg, Martinistrasse 52, D-2000 Hamburg 20 (FRG)

Presented April 1985

The monoclonal antibody 486 P 3/12 (IgM $\lambda$ ) raised against the bladder carcinoma line $486 \mathrm{P}$ detects a 220-kilodalton glycopro-tein expressed on 17/19 bladder carcinomas tested so far. This antigen is expressed fo-cally by a few single superficial cells in the normal human bladder mucosa.

We used 486 P 3/12 to identify tumor cells in bladder washings from four groups of patients. Group I consisted of 40 patients with histologically proven bladder carcinomas. Group II consisted of 41 age-matched patients with no bladder disease $(n=24)$ and children with significant bacteriuria $(n=17)$. Group III consisted of 41 patients who received after complete removal of their bladder tumor mitomycin $\mathrm{C}$ instillation therapy and were disease-free as assessed by endos-copy. Group IV consisted of 39 patients whose bladder tumors were completely removed at least 3 months earlier and who had no evidence of local tumor recurrence.

The cytocentrifuged specimens were analyzed by immunocytology using alkaline phosphataseconjugated indicator anti-

bodies and standard cytology. Immunocytology was analyzed quantitatively. In the control group (group II), none of the patients had more than 25\% immunocytologically positive cells in the bladder washings using $486 \mathrm{P}$ 3/12. All specimens were regarded as immunocytologically negative with less than $25 \%$ of reactive cells. Those with more than $30 \%$ reactive cells were defined as immunocytologically positive.

With this baseline, 36 out of 40 patients (90\%) with bladder tumors (group I) yielded positive immunocytologic findings. The overall positive rate using standard urinary cytology was $47 \%$. The highest false negative rate was observed in grade I and grade II tumors. Comparing both techniques, the sensitivity for detecting bladder cancer cells in the urine could be improved by $97.5 \%$ in group I using immunocytology instead of standard cytology methods. 